\title{
Study of the effect of external noise pickups on the performance of a cryogenic bolometer
}

\author{
A. Garai, ${ }^{1,2}$ A. Reza, ${ }^{3, a)}$ A. Mazumdar,,${ }^{1,2}$ H. Krishnamoorthy, ${ }^{1,2}$ G. Gupta,${ }^{3}$ \\ M.S. Pose,${ }^{3}$ S. Mallikarjunachary, ${ }^{3}$ V. Nanal, ${ }^{3}$ R. G. Pillay, ${ }^{3, b)}$ and \\ S. Ramakrishnan ${ }^{4}$
}

${ }^{1}$ India-based Neutrino Observatory, Tata Institute of Fundamental Research, Homi Bhabha Road, Mumbai 400005, India

${ }^{2}$ Homi Bhabha National Institute, V.N. Purav Marg, Anushaktinagar, Mumbai 400094, India

${ }^{3}$ Department of Nuclear and Atomic Physics, Tata Institute of Fundamental Research, Homi Bhabha Road, Mumbai 400005, India

${ }^{4}$ Deptment of Condensed Matter Physics \& Materials Science, Tata Institute of Fundamental Research, Homi Bhabha Road,Mumbai 400005, India

\author{
a)Electronic mail: ashifreza86@gmail.com \\ b)Present address: Department of Physics, Indian Institute of Technology Ropar, Rupnagar - 140001, India
}

\begin{abstract}
This paper reports the detailed noise characterization, investigation of various noise sources and its mitigation to improve the performance of a cryogenic bolometer detector. The noise spectrum has been measured for a sapphire bolometer test setup with indigenously developed NTD Ge sensor in the CFDR system at Mumbai. The effect of external noise, arising either from ground loops in the system or from the diagnostic and control electronics of the cryostat, on the performance of a cryogenic bolometer is assessed. A systematic comparison of the influence of different noise pickups on the bolometer resolution is also presented. The best-achieved resolution $\left(\sigma_{\mathrm{E}}\right)$ at $15 \mathrm{mK}$ is $\sim 15 \mathrm{keV}$ for heater pulses and appears to be mainly limited by the noise due to the pulse tube cryocooler.
\end{abstract}

Over the last few decades, there is a strong interest in application of cryogenic bolometers in rare decay studies due to their excellent energy resolution, high sensitivity and a possibility to upscale the size ${ }^{1-2}$. Generally, the performance of cryogenic bolometers is not limited by the intrinsic resolution, but by the external noise sources in the system $^{3}$. To achieve operating temperatures of $5-10 \mathrm{mK}$, the pulsed tube based cryogenic systems, are attractive for long term operations in underground laboratories. In these systems, noise induced due to the mechanical vibrations is a major concern and attempts to reduce the same have been reported ${ }^{4-5}$. Other significant contributions arise from the $\mathrm{mK}$ thermometry (sensor+readout electronics) and system specific components like vacuum pumps and control units. In addition, minimization of ground loops is essential. With this motivation, we have studied the noise spectra of a test bolometer in the cryogen free dilution refrigerator.

The CFDR-1200 (Leiden Cryogenics) has been set up ${ }^{6}$ at Tata Institute of Fundamental Research, Mumbai, for the prototype development of a Sn cryogenic bolometer (TIN.TIN -The INdia based TIN detector) ${ }^{7}$. To minimize the pulse tube vibration, a linear drive unit is used to smoothen the movement of motorized rotary valve control unit. The mixing chamber temperature $\left(T_{\mathrm{MC}}\right)$ is monitored by a Carbon Speer sensor, calibrated against a Cerium Magnesium Nitrate (CMN) thermometer. The CFDR setup is enclosed within a Faraday Cage to reduce the effect of EMI. The valve control unit of the pulse tube cooler is kept detached from the cryostat body to minimize the induced vibration. Presently, a test bolometer setup is developed comprising a sapphire absorber $(20 \mathrm{~mm} \times 20 \mathrm{~mm} \times 0.4 \mathrm{~mm})$, indigenously fabricated NTD Ge sensor ${ }^{8-9}$ and a heater element, which are strongly coupled to the absorber using a low-temperature Araldite. The absorber is weakly connected to the $\mathrm{Cu}$ block using $0.1 \mathrm{~mm}$ thick Araldite dots (dia 1mm). The heater is developed by evaporating a $0.2 \mu \mathrm{m}$ thick $\mathrm{Au}$ meander on a $\mathrm{Si}$ substrate. The heater resistance, measured using an AC resistance bridge AVS$47 \mathrm{~B}$ (PICOWATT), is $\sim 0.6 \mathrm{k} \Omega$ at $1 \mathrm{~K}$ and remains constant down to $10 \mathrm{mK}$. The NTD Ge sensors $(6 \mathrm{~mm} \times 3 \mathrm{~mm} \times 1 \mathrm{~mm})$ are developed in-house. Measurements of the resistance of NTD Ge sensor $\left(R_{\mathrm{S}}\right)$ are done with AVS-47B for $R_{\mathrm{S}}<2 \mathrm{M} \Omega$. At lower temperatures, (i.e. $R_{\mathrm{S}}>2 \mathrm{M} \Omega$ ), $R_{\mathrm{S}}$ is measured by applying a pseudoconstant current, generated by the voltage $V_{\mathrm{B}}$ through a high resistor $\left(R_{\mathrm{B} 1}=20 \mathrm{G} \Omega\right)$, connected in series with the sensor (Fig. 1).

The resistance $\left(R_{\mathrm{S}}\right)$ for the NTD Ge Sensor (DB27) has been measured in the range of $10-400 \mathrm{mK}$. It was observed that the measured resistance showed a deviation from the Mott behaviour ${ }^{10}$ below $50 \mathrm{mK}$ and saturated at $250 \mathrm{M} \Omega$, even though $T_{\mathrm{MC}}$ cools down to $10 \mathrm{mK}$. Therefore, noise spectra have been recorded to understand the effect of various external noise sources.

One of the major factors limiting the bolometer performance can be inefficient grounding leading to several ground loops. To overcome this, ground 


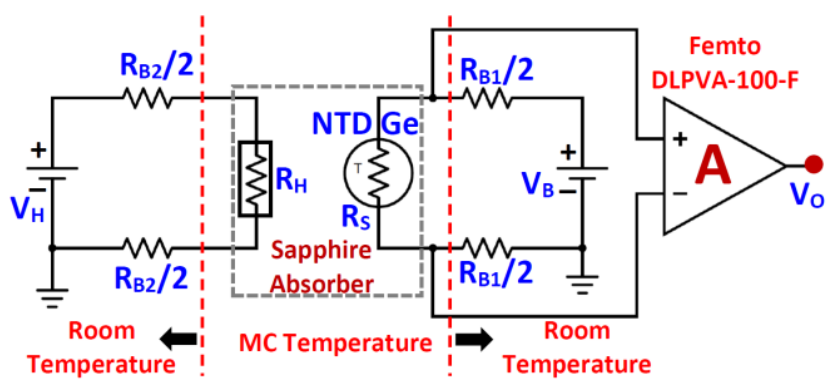

FIG. 1. Schematic circuit for the sapphire bolometer readout.

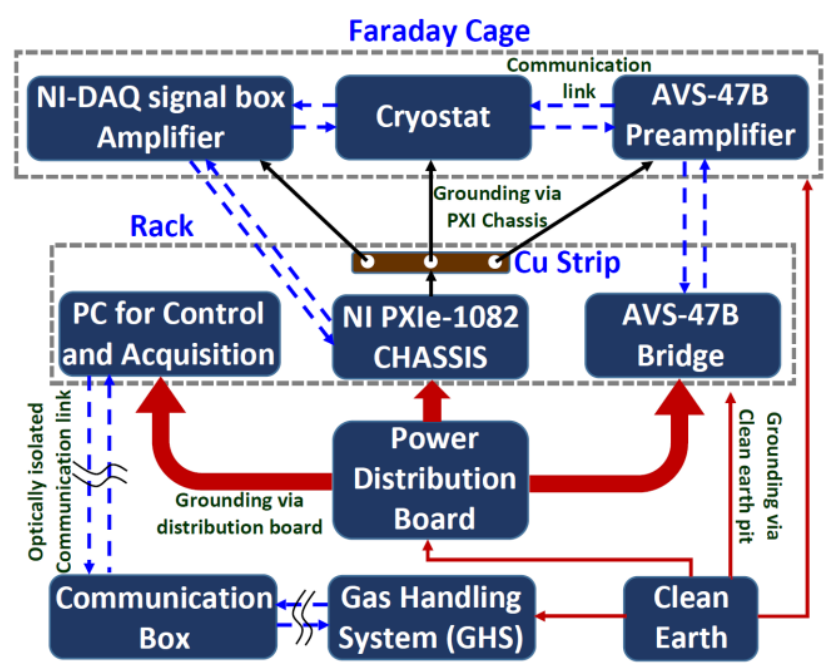

FIG. 2. A schematic layout of optimized ground configuration.

connections to various units are fanned out from a specially designated clean earth pit. The chassis acts as a master ground for the DAQ box, the amplifier, the AVS preamplifier as well as the cryostat (see Figure 2). The CFDR cryostat, the differential amplifier and DAQ signal box, AVS preamplifiers for diagnostic thermometry and the sensor readout are enclosed within the Faraday Cage. The CFDR controls for ${ }^{3} \mathrm{He}-{ }^{4} \mathrm{He}$ gas handling system and accessories are routed through an optically isolated USBRS232 interface. The NI-PXI chassis, AVS bridges and PCs are fitted in an anodized metallic rack. The PXI is connected to the PC with an optical link to reduce the EMI pickups.

As an example of a ground loop in the sub-optimal grounding, the master ground is assigned to the cryostat, which results in multiple ground connections for DAQthrough the cryostat and through the power connection. In this configuration, $50 \mathrm{~Hz}$ and its harmonics are clearly visible in the noise spectra (Fig. 3a). As a result, the $T_{\mathrm{MC}}$ could not be stabilized below $15 \mathrm{mK}$.

Further noise measurements have been carried out with optimal grounding at $T_{\mathrm{MC}}=10 \mathrm{mK}$ for four configurations: Case-I: pulse tube in normal drive, motor head mounted on the cryostat; Case-II: pulse tube in linear drive, motor head detached from the cryostat; Case-III: Case-II configuration with gauge electronics of Inner Vacuum Chamber disconnected; Case-IV: Case-II configuration with electronics of all vacuum gauges
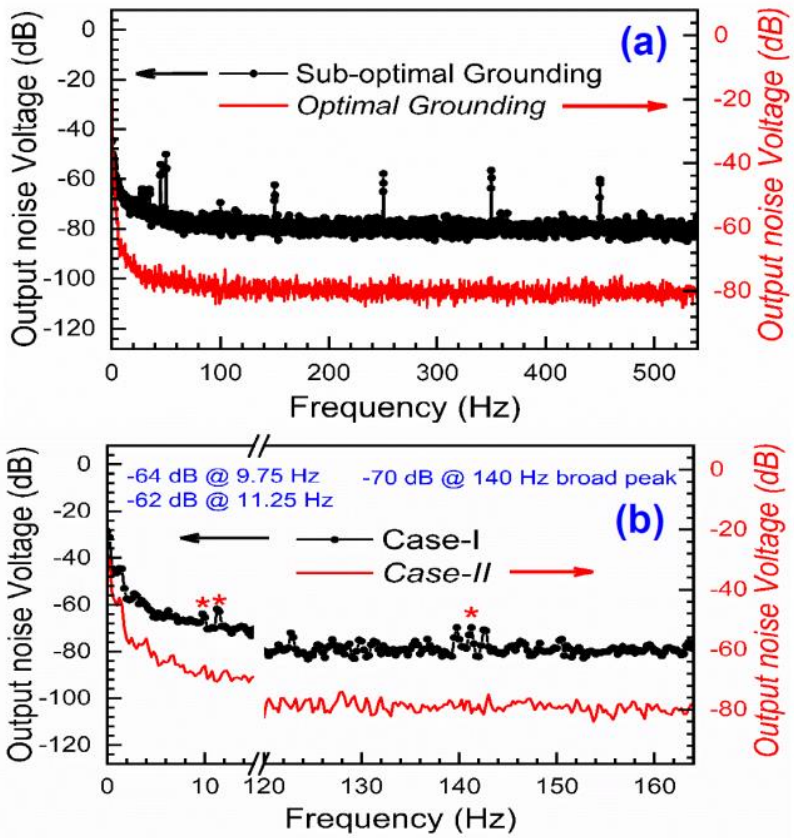

FIG. 3. FFT spectra of DB27 output showing (a) Effect of ground loops on $50 \mathrm{~Hz}$ noise at $T_{\mathrm{MC}}=15 \mathrm{mK}$ (b) Effect of pulse tube induced noise.

disconnected. In all four cases, noise spectra are measured over a frequency range of $0-25 \mathrm{kHz}$ with a voltage gain of $80 \mathrm{~dB}$. Noise peaks at $9.75 \mathrm{~Hz}, 11.25 \mathrm{~Hz}$ and $140 \mathrm{~Hz}$ seen in Fig. $3 \mathrm{~b}$ for Case-I are mainly contributed by the harmonics of pulse tube induced noise of $1.4 \mathrm{~Hz}$, which disappears for linear drive mode in Case-II. This helps in cooling the sensor, which is also reflected in the measured resistance of $370 \mathrm{M} \Omega$ as compared to $250 \mathrm{M} \Omega$ for Case-I. However, no appreciable difference in the FFT spectra above $500 \mathrm{~Hz}$ is observed between Case-I and Case-II. Vacuum pumps and gauges are found to contribute to high frequency pickups at $827 \mathrm{~Hz}, 985.5 \mathrm{~Hz}$ and $16 \mathrm{kHz}$. Details of the various noise pickups are summarized in Table I. In Case$\mathrm{IV}$, the measured resistance is $594 \mathrm{M} \Omega$.

Figure 4(a) shows that the sensor resistance has increased substantially in Case-IV as compared to Case-I and the sensor follows Mott behavior down to $40 \mathrm{mK}$ indicating a net improvement in reducing the heat leak to the sensor. The effect of ground loops was also reflected in the minimum $T_{\mathrm{MC}}$ and in the measured resolution. For Case-IV, $T_{\mathrm{MC}} \sim 5 \mathrm{mK}$ could be achieved as compared to $6.7 \mathrm{mK}$ in the sub-optimal case.

TABLE. 1. Output voltage in $\mathrm{dB}$ (text in italics) for different frequencies as measured from the noise spectra. (BBN: Below Baseline Noise)

\begin{tabular}{c|c|c|c|c|c}
\hline \hline \multirow{2}{*}{\begin{tabular}{c} 
Different \begin{tabular}{c} 
Fases \\
\cline { 2 - 6 }
\end{tabular} \\
\cline { 2 - 6 }
\end{tabular}} & $\mathbf{9 . 7 5 / 1 1 . 2 5 / 1 4 0}$ & $\mathbf{5 4 3 . 5}$ & $\mathbf{8 2 7 . 5}$ & $\mathbf{9 8 5 . 5}$ & $\begin{array}{c}\mathbf{1 5 . 7 3 k} \\
\mathbf{1 6 . 2 7} \mathbf{k}\end{array}$ \\
\hline Case-I & $-64 /-62 /-70$ & -63 & -64 & -62 & -55 \\
\hline Case-II & $B B N$ & -62.5 & -65.7 & -58.3 & -59 \\
\hline Case-III & $B B N$ & -62 & -70.4 & -60.4 & $B B N$ \\
\hline Case-IV & $B B N$ & -63 & -71.6 & -60.2 & $B B N$ \\
\hline \hline
\end{tabular}



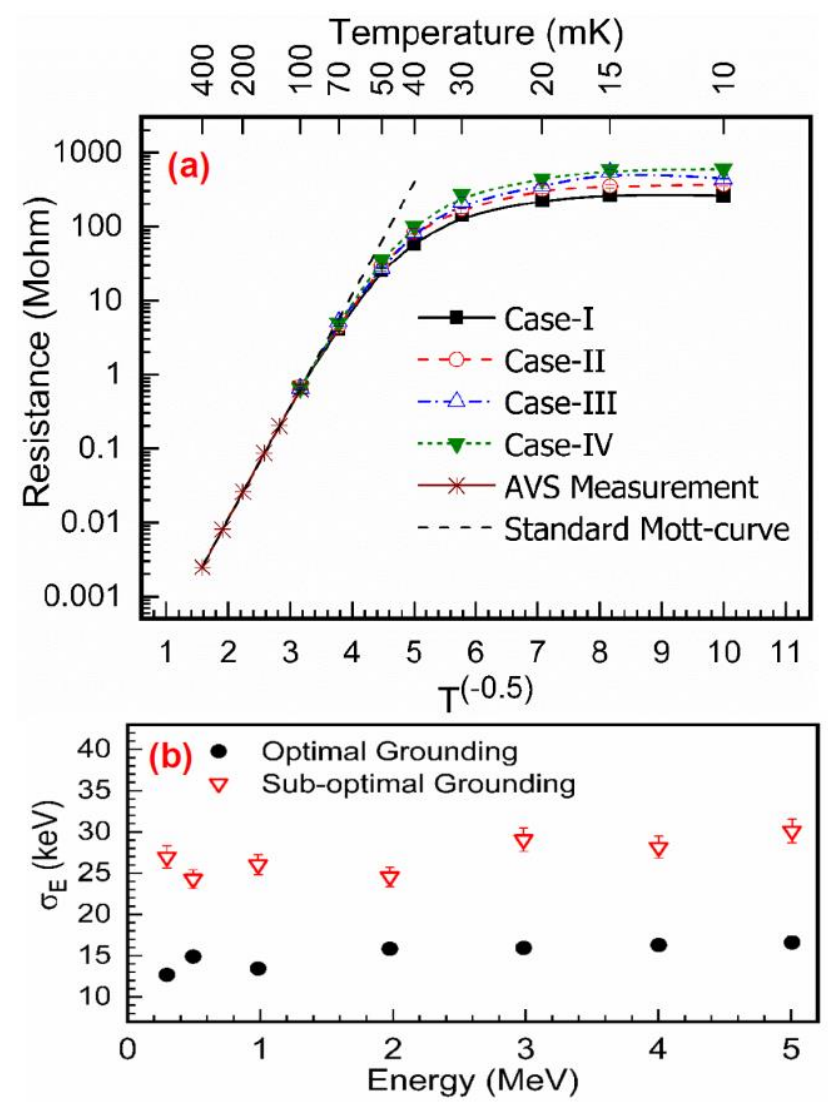

FIG. 4. (a) Measured resistance of DB27 for $\mathrm{T}=10-400 \mathrm{mK}$ together with standard Mott curve fit $\left(R_{0}=10.2(0.5) \Omega\right.$ and $\left.T_{0}=12.2(0.2) \mathrm{K}\right)$ (b) Measured bolometer resolution with the phonon signal.

To measure the bolometer resolution, an external phonon signal is generated, by connecting a $2 \mathrm{M} \Omega$ resistor $\left(R_{\mathrm{B} 2}\right)$ in series with the heater (Fig. 1). The pulses of varying amplitude, $200 \mu$ s wide with a repetition rate of $2 \mathrm{~Hz}$, are supplied to the heater to simulate phonon signal. The voltage signal from the sensor is analyzed off-line ${ }^{11}$. Figure 4(b) shows that the measured resolution at $15 \mathrm{mK}$ for Case-IV, which worsens by $\sim 80 \%$ in case of the suboptimal grounding. The resolution is found to improve by $\sim 30 \%$ when the pulse tube is switched from normal drive to linear drive. However, different configurations (Case-II to Case-IV) yield similar results $(\sim 15 \mathrm{keV})$ within measurement errors.

In summary, we have presented the influence of various noise sources on the performance of a sapphire bolometer in the CFDR system inclusive of control, diagnostic thermometry and DAQ. It is shown that the presence of ground loops can worsen the bolometer resolution $\sim 80 \%$. Further, the noise pickups from vacuum pumps and gauges also introduce thermal load on the sensor. In the best configuration, $\sigma_{\mathrm{E}} \sim 15 \mathrm{keV}$ is obtained for the sapphire bolometer at $15 \mathrm{mK}$.

The authors would like to thank TIN.TIN and INO collaboration for their support and Mr. K.V. Divekar for assistance during measurements.
${ }^{3}$ C. Enss et al., J. Low Temp. Phys. 151, 5 (2008).

${ }^{4}$ A. M. J. den Haan et al., Rev. Sci. Instrum. 85, 035112 (2014).

${ }^{5}$ A. D'Addabbo et al., Cryogenics 93, 56 (2018).

${ }^{6} \mathrm{~V}$. Singh et al., Pramana 81, 719 (2013).

${ }^{7}$ V. Nanal, EPJ Web Conf. 66, 08005 (2014).

${ }^{8}$ S. Mathimalar et al., 11th International Workshop on Low Temperature Electronics, July, 7 - 9, France (2014).

${ }^{9}$ A. Garai et al., J. Low Temp. Phys. 184, 609 (2016).

${ }^{10} \mathrm{E}$. Pasca et al., Proceedings of 8th International Conference on Advanced Technology and Particle Physics, 2, 93 (2004).

${ }^{11} \mathrm{~A}$. Garai et al., Proceedings of DAE International Symposium on Nuclear Physics, 63, 1140 (2018). 